\title{
Analyzing recognition performance with sparse data
}

\author{
Ching-Fan Sheu, Yuh-Shiow Lee, and Pei-Ying ShiH \\ National Chung Cheng University, Chia-Yi, Taiwan
}

\begin{abstract}
Experiments in which recognition performance is measured sometimes involve only a small number of observations per subject, rendering $d^{\prime}$ analysis unreliable (Schooler \& Shiffrin, 2005). Here, we introduce, in signal detection models, subject-specific random variables to account for heterogeneous hit and false alarm rates among individuals. Population $d^{\prime}$ effects for comparing groups are estimated, in this approach, by pooling information from a sample of subjects across experimental conditions. The method is validated by a simulation study and is illustrated with an analysis of the effect of neutral and emotional words on recognition performance, employing the emotional Stroop task (Lee \& Shih, 2007).
\end{abstract}

This article presents a statistical method for analyzing recognition performance with sparse data. The problem can be stated as follows: How can we properly estimate $d^{\prime}$ (or any other model parameters of signal detection theory) from experiments in which only a small number of stimulus trials are available for presentation, resulting in unreliable hit and false alarm rates? Since the parameters of signal detection models are the quantities commonly used to characterize recognition performance, without reliable estimates of these parameters comparisons of experimental effects between conditions will not be possible. Thus, it is important to have a solution to this problem since experimental paradigms, such as the Deese/RoedigerMcDermott (DRM) false memory paradigm (Roediger \& McDermott, 1995) and the emotional Stroop task (Gotlieb \& McCann, 1984), typically preclude the possibility of collecting a large number of observations per subject.

One way to compensate for the short series of observations per subject is to increase the number of subjects in a condition and to devise a way to aggregate information between individuals so that reliable measures of recognition memory performance can be obtained. For example, one might simply aggregate the hit and false alarm rates of all the subjects in an experimental condition and calculate bootstrap confidence intervals (Efron \& Tibshirani, 1991) for aggregating $d^{\prime}$ values to test the differences in recognition performance between experimental conditions. This is essentially what was proposed by Schooler and Shiffrin (2005) for measuring recognition performance with sparse data. However, their method has only limited usefulness for three reasons. First, it assumes that hit and false alarm rates in a condition must be homogeneous, when it is more likely that genuinely different response patterns exist among individuals. Second, it is applicable for testing hypotheses under the restrictive assumptions that signal and noise have the same variance and are normally distributed. These assumptions can, of course, be relaxed. But the bootstrap procedure will have to be retooled. Third, the method lacks a formal mechanism for linking parameters of detection analysis directly to experimental conditions, as is shown, for example, in Sheu and Heathcote (2001). Consequently, the bootstrap procedure needs to be modified each time the details of experimental conditions change.

By contrast, the method presented here extends the well known connection between signal detection models and generalized linear models (DeCarlo, 1998; Sheu \& Heathcote, 2001) by (1) including random effects to account for variable hit and false alarm rates between subjects and (2) relaxing the distribution and equal-variance assumptions for signal and noise presentations. The resulting formulation is a class of nonlinear mixed-effects models (Davidian \& Giltinan, 1995) whose parameters can be estimated using standard routines implemented in general purpose statistical software packages, such as SAS (SAS Institute, 2004) and R (R Development Core Team, 2006).

The underlying premise of this approach is that subjects respond differently: Some are high responders, some are low responders, and some are medium responders. In other words, individuals are assumed to have their own subject-specific mean hit and false alarm rates for a condition, thereby generating heterogeneous recognition performance. The same idea can also be put forth to argue for a distribution of sensitivity for a population of subjects. Analogous to the mixed-effects ANOVA, our formulation assumes a normal distribution for $d^{\prime}$ (or subject-specific random criteria) in the population and adjusts model parameter estimates as a function of both between- and within-subjects variabilities in hit and false alarm rates. Unlike the bootstrap method of Schooler and Shiffrin

C.-F. Sheu, psycfs@ccu.edu.tw 
(2005), our procedure directly estimates the parameters of the signal detection model under consideration so that the investigators can examine whether an experimental manipulation is better accounted for by a location shift in sensitivity or by response bias.

The purpose of this article is to illustrate the generality and flexibility of our approach to estimating parameters of signal detection models for recognition performance with sparse data. The estimation procedures will be implemented using SAS PROC NLMIXED (Wolfinger, 1999). The generality of this approach is demonstrated by the ease with which this framework can accommodate different distribution assumptions and performance measures, such as the area under the receiver operating characteristic curve.

This article is organized as follows. The next section formulates a mixed-effects signal detection model for dichotomous responses in the context of recognition memory. The third section reports a simulation study similar to those performed in Schooler and Shiffrin (2005) to assess how well model parameters can be recovered by the estimation procedure. The fourth section illustrates the method with a signal detection analysis of an emotional Stroop recognition task (Lee \& Shih, 2007). The final section discusses implications and potential applications.

\section{Mixed-Effects Signal Detection Model}

The theory of signal detectability was originally proposed as a way to model data from detection and discrimination in perceptual experiments (Peterson, Birdsall, \& Fox, 1954; Tanner \& Swets, 1954). A subject is confronted with an observation in which a (weak) signal may or may not be embedded in noise and is required to make a binary choice $($ yes-no). With identical signal intensity, the chance of a correct detection is influenced by the observer's decision criterion, which cannot be observed. The experimenter can, however, induce the observer to change his/ her criterion by instructions or by manipulating payoff.

Researchers soon realized that signal detection theory is also applicable to the study of human memory (Banks, 1970). For example, in the study phase of a recognition experiment, lists of words are presented to subjects. In the test phase, the subjects are asked to judge whether words on a new list are old (signal) or new (noise). A hit occurs when the subject responds to the presentation of an old word by correctly identifying that it is old; a false alarm occurs when the subject incorrectly identifies a word as being old when, in fact, it is new. The standard models of signal detection theory assume that the hit and false alarm probabilities arise from the tails of corresponding signal and noise distributions given a common threshold, $c$. The distribution functions are usually taken to be either normal (Green \& Swets, 1966) or logistic (Luce, 1963).

In the normal case, the noise distribution is usually assumed to be a standard normal distribution, and the signal distribution is a normal distribution with a mean of $\mu_{\mathrm{s}}$ and a standard deviation $\sigma_{\mathrm{s}}$. The hit and false alarm probabilities, $\pi_{\mathrm{s}}$ and $\pi_{\mathrm{n}}$, are transformed by $\Phi^{-1}$, the inverse distribution function of a standard normal distribution:

$$
\Phi^{-1}\left(\pi_{\mathrm{n}}\right)=-c
$$

and

$$
\Phi^{-1}\left(\pi_{\mathrm{s}}\right)=\frac{\mu_{\mathrm{s}}-c}{\sigma_{\mathrm{s}}} .
$$

When the variances for signal and noise distributions are assumed to be the same, the only parameter remaining in the model is the famous measure of detectability:

$$
d^{\prime}=\Phi^{-1}\left(\pi_{\mathrm{s}}\right)-\Phi^{-1}\left(\pi_{\mathrm{n}}\right) .
$$

For the mixed-effects signal detection model, we assume a random variable $\mathbf{b}_{i}$ for each subject $i$ and write

$$
\Phi^{-1}\left(\pi_{i X_{j}}\right)=\sigma_{\mathrm{s}}^{-X_{j}}\left[\mu_{\mathrm{s}} X_{j}-\left(c+\mathbf{b}_{i}\right)\right],
$$

where $X_{j}$ is an indicator variable for a signal presentation at trial $j$. The random variable $\mathbf{b}_{i}$ is assumed to have a normal distribution with zero mean and a variance of $\sigma^{2}$, which represents between-subjects variation. In other words, people (in the same condition) are assumed to possess the same sensitivity but varying criteria. This is not incompatible with the practice that, when individual data are rich enough, one usually extracts individual sensitivity estimates without simultaneously holding a set of common criteria for all individuals and that these individual sensitivity estimates are thought of as realizations of a population parameter.

The distinctive feature of this model is that the mean hit and false alarm rates (on the probit scale) are modeled as a combination of population characteristics shared by all individuals and subject-specific effects that are unique to a particular individual. It captures two notions: (1) Repeated observations on the same subject have the same hit and false alarm probabilities, and (2) hit and false alarm rates vary from one individual to another in the population. The presence of a subject-specific random effect allows the estimation of location shift (or $d^{\prime}$ ) to benefit from an increased number of subjects even though each individual may contribute only a small number of observations.

The data analyst can, of course, extend the current formulation to treat some or all parameters in a signal detection model as random effects. However, as the number of random effects increases in a model, the estimation can become numerically unstable or even computational intractable. Graphing the data or checking residuals can usually help a data analyst select better models. With sparse data, however, plotting is often unilluminating. Other things being equal, sparse data tend to favor simple over complicated models.

The ability of mixed-effects models to account for data from a sample of individuals across different experimental conditions represents an important generalization of signal detection analysis. Such generalization is similar in spirit to the hierarchical structure of Bayesian formulations of signal detection models (Sheu, 1998). We note that when the variances for signal and noise are assumed to be the same, the mixed-effects formulation reduces to what are known as generalized linear mixed models (McCulloch \& Searle, 2001).

Parameter estimation. Ogilvie and Creelman (1968) developed classical maximum-likelihood estimation procedures for the logit model. Dorfman and Alf (1969) 
solved the same estimation problem under the normal distributional assumption. Sheu and Heathcote (2001) used a nonlinear regression approach to estimate signal detection model parameters. All of these solutions used modefinding algorithms, such as Newton-Raphson, to locate a value of the parameter that maximizes the likelihood function. Sheu (1998), on the other hand, presented a Markov chain Monte Carlo simulation approach to estimating the parameters of signal detection theory.

To fit the mixed-effects signal detection model, we use the NLMIXED procedure (SAS Institute, 2004; Wolfinger, 1999), which directly fits the model by maximizing an approximation to the likelihood integrated over the random effects. An adaptive version of Gauss-Hermite quadrature (Abramowitz \& Stegun, 1972) is used to approximate the likelihood, and the default maximization routine is a dual quasi-Newton algorithm (Pinheiro \& Bates, 1995). A more detailed discussion of parameter estimation using the NLMIXED procedure can be found in Sheu, Chen, Su, and Wang (2005) in the context of fitting item response theory models.

\section{Simulations}

To validate the implementation of the mixed-effects signal detection models in the NLMIXED procedure, we simulate observations on the basis of a normal, unequalvariance signal detection model for recognition memory and examine how well parameter values can be recovered. The design of our simulation study is similar in spirit to that of Schooler and Shiffrin (2005), who, in turn, generated their conditions to model the DRM paradigm.

Table 1 presents the model parameters and resulting hit and false alarm probabilities of a hypothetical recognition memory experiment. There are a total of six conditions with two different values for $\mu_{\mathrm{s}}$ (differing by 1) crossed with three different values for criterion $c$, controlled by the bias $k=-1,0,1$. A constant signal variance $\sigma_{\mathrm{s}}$ is used for all six conditions. The noise variance is, by default, set to 1 . Columns 6 and 7 of Table 1 show the corresponding hit and false alarm probabilities of the true signal detection model described in columns 2-5.

For signal trials in each condition, we draw a random number from the uniform distribution between 0 and 1 and check whether it is smaller than the hit rate for that condition. The observation is scored as a hit if it is; otherwise, it is scored as a miss. Similarly, for noise trials in each condition, we draw a random number from the

Table 1

The Six Conditions Used to Simulate Recognition Performance

\begin{tabular}{ccccrcc}
\hline Condition & $\mu_{\mathrm{s}}$ & $\sigma_{\mathrm{s}}$ & $c=\frac{\mu_{\mathrm{s}}}{2}+k$ & \multicolumn{1}{c}{$k$} & Hit & FA \\
\hline 1 & 2.5 & $\sqrt{2}$ & 0.25 & -1 & .944 & .401 \\
2 & 2.5 & $\sqrt{2}$ & 1.25 & 0 & .812 & .106 \\
3 & 2.5 & $\sqrt{2}$ & 2.25 & 1 & .570 & .012 \\
4 & 3.5 & $\sqrt{2}$ & 0.75 & -1 & .974 & .227 \\
5 & 3.5 & $\sqrt{2}$ & 1.75 & 0 & .892 & .040 \\
6 & 3.5 & $\sqrt{2}$ & 2.75 & 1 & .702 & .003 \\
\hline
\end{tabular}

Note-A description of the underlying normal signal detection model for each condition is provided in the text. FA, false alarm.
Table 2

Parameter Estimates and Standard Errors Obtained From Long and Short Series Simulation Runs

\begin{tabular}{cccc}
\hline Parameter & Expected & $\begin{array}{c}\text { Long Series } \\
\text { Est. } \pm S E\end{array}$ & $\begin{array}{c}\text { Short Series } \\
\text { Est. } \pm S E\end{array}$ \\
\hline$\mu_{\mathrm{s}}$ & 3.50 & $3.721 \pm 0.095$ & $3.690 \pm 0.095$ \\
$d$ & 1.00 & $1.123 \pm 0.064$ & $1.100 \pm 0.064$ \\
$\sigma_{\mathrm{s}}$ & $\sqrt{2}$ & $1.478 \pm 0.056$ & $1.515 \pm 0.058$ \\
$c_{1}$ & 0.25 & $0.267 \pm 0.023$ & $0.244 \pm 0.023$ \\
$c_{2}$ & 1.25 & $1.267 \pm 0.028$ & $1.248 \pm 0.028$ \\
$c_{3}$ & 2.25 & $2.309 \pm 0.054$ & $2.328 \pm 0.055$ \\
$c_{4}$ & 0.75 & $0.751 \pm 0.025$ & $0.747 \pm 0.025$ \\
$c_{5}$ & 1.75 & $1.813 \pm 0.039$ & $1.745 \pm 0.038$ \\
$c_{6}$ & 2.75 & $2.944 \pm 0.075$ & $2.903 \pm 0.074$ \\
$\sigma$ & - & $0.004 \pm 0.051$ & $0.103 \pm 0.023$ \\
\hline
\end{tabular}

Note-Column 2 gives the true parameter values of the signal detection model. The parameter $d$ is the difference between the value of the location parameter for Conditions 1,2, and 3 and that of the same parameter for Conditions 4, 5, and 6 .

uniform distribution between 0 and 1 and check whether it is smaller than the false alarm rate for that condition. The observation is scored as a false alarm if it is; otherwise, it is scored as a correct rejection.

We performed three different simulations. For what is called the long series, we simulated 600 signal and 600 noise trials for each of 5 (virtual) subjects for each condition. For the so-called short series, we generated 5 signal and 5 noise trials for each of 600 subjects for each condition. One might compare the long series cases with psychophysical experiments and the short series with psychometric evaluations such as the Graduate Record Examination. In addition to examining whether or not the true parameter values are recovered, the estimated variance of the random effect $\sigma^{2}$ was checked to see whether it was more prominent for the short series, since between-subjects variability was expected to be greater in this case than in the long series, where a small number of subjects performed a large number of trials. In these simulations, however, the estimated value for $\sigma^{2}$ should be small, since the true model was a fixed-effects model and the only source of variability was the random error introduced by the simulation itself.

The results of both the long and the short series simulation runs are presented in Table 2. The parameter estimates obtained from either series are quite close to their corresponding expected values in the true model. In terms of standard deviation, the between-subjects variability for the short series is estimated to be about a factor of 25 larger than that for the long series.

The third simulation is what we shall call the regular series, in which three signal and three noise trials for each of 36 subjects were generated for each condition. This is a more realistic scenario for memory experiments employing sparse data paradigms. The smallest number of signal (or noise) trials used in Schooler and Shiffrin's (2005) study was also three. On the other hand, the number of subjects was chosen to be the same as that in a real data example to be discussed later.

Unlike the case for either the long or the short series, in which only 1 run was performed, 20 runs for the regular series were replicated in order to examine the variability 
of parameter estimates across simulation runs. The accuracy of parameter estimates were assessed in two ways: the average of the differences between the estimated values and the true value, which is known as bias, and the root-mean-square error (RMSE), which is a measure of accuracy that combines bias and the standard error of the estimator. Their formulas are given below for the parameter $\theta$, its estimator, $\hat{\theta}$, and the number of the latter's realizations, $N$ :

$$
\operatorname{bias}=\frac{1}{N} \sum_{i=1}^{N}\left(\hat{\theta}_{i}-\theta\right)
$$

and

$$
\mathrm{RMSE}=\sqrt{\frac{1}{N} \sum_{i=1}^{N}\left(\hat{\theta}_{i}-\theta\right)^{2}} .
$$

The results for the regular series are shown in Figure 1 and Table 3 . In only 1 out of 20 runs, the approximate $95 \%$ confidence interval for model parameters failed to cover their true values. The accuracies of the parameter estimates, as assessed by the bias and RMSE measures, are similar to those reported by Murphy and Dunne (2005), in which mixed-effects and fixed-effects modeling with intersubject variability was examined using a logit-normal model (akin to the probit-normal model considered here) for binary responses.

\section{Emotional Stroop Recognition Task}

Lee and Shih (2007) investigated the effect of neutral and emotional words on memory, using the Stroop colornaming task (Gotlieb \& McCann, 1984). Thirty-six Taiwanese college students studied 20 words and then were tested for recognition of 40 words. These included four types of words: neutral words and three types of emotional

\section{Approximate 95\% Confidence Intervals}

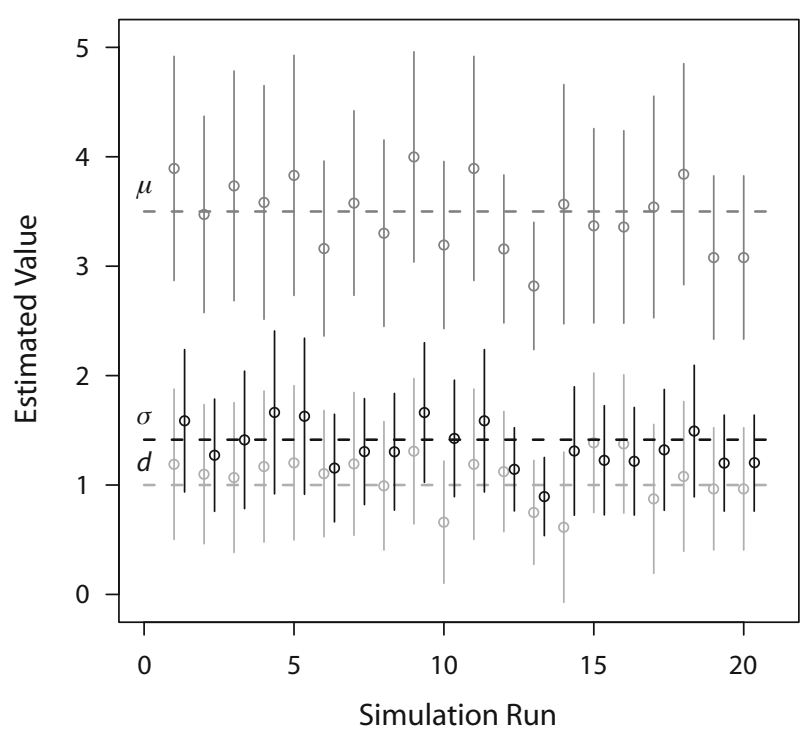

Figure 1. The approximate $95 \%$ confidence intervals constructed for the true model parameters $\mu_{\mathrm{s}}, d$, and $\sigma_{\mathrm{s}}$, based on each of the 20 simulation runs.
Table 3

The Estimates of Bias and Root-Mean-Square Error (RMSE) Obtained From 20 Simulation Runs of the Regular Series

\begin{tabular}{ccc}
\hline Parameter & \multicolumn{1}{c}{ Bias } & RMSE \\
\hline$\mu_{\mathrm{s}}$ & -0.028 & 0.322 \\
$D$ & 0.065 & 0.219 \\
$\sigma_{\mathrm{s}}$ & -0.064 & 0.209 \\
\hline
\end{tabular}

words (negative high, negative, and positive). For each word type, Chinese words, matched in word frequency, number of strokes, familiarity, and concreteness, were selected. The matching of stimulus word type severely reduced the number of words available for presentation. The number of signal as well as noise trials was five, a total of 10 Chinese words for each word type. As a consequence, the observed hit and false alarm rates for several subjects in some conditions were one or zero, rendering reliable $d^{\prime}$ analysis infeasible.

To compare recognition performance for neutral words against the three emotional word types, we fit a series of mixed-effects signal detection models to hit and false alarm rates and compared the resulting $d^{\prime}$ estimates. We use Akaike's information criterion (Akaike, 1974) and Schwarz's (1978) Bayesian information criterion to compare these models. These information criteria penalize models with additional parameters. The results of a simple model, which assumed that signal and noise have the same variance and that the decision criterion remained invariant for all conditions, are displayed in Table 4. An inspection of the $d^{\prime}$ estimates showed that, as compared with neutral stimuli, memory for words is affected more by negative than by positive valences of the words. Note that the estimated value of $\sigma$ is much larger than those found in the simulations, in which the true model is a fixed-effect model. The fact that the value of $\sigma$ is statistically different from zero suggests that introducing a subject-specific random effect in the signal detection analysis was useful in capturing the heterogeneous hit and false alarm rates. In contrast, a crude $d^{\prime}$ analysis based on aggregate hit and false alarm rates failed to detect any differences in recognition performance between the emotional and the neutral words.

The assumption that criterion is invariant received support from a study by Ben-David, Calderon, and Algom (2005), in which a severe time limit on reporting was imposed to induce relatively high error rates so that the emotional Stroop effect would become amenable to standard signal detection analysis.

Listing 1 presents the SAS code segment used to analyze the emotional Stroop data. The readers may consult the article by Sheu et al. (2005) for a quick start on how to implement mixed-effects models in PROC NLMIXED. A brief explanation of the statements in Listing 1 is provided here.

The data step reads in the data file, emostroop.dat, and sets up input variables, each of which matches the corresponding position in the columns of the data file. Each row of the data file consists of values for the following variables: subject identification, treatment indicator ( 1 for signal trial and 0 for noise trial), the number of yes re- 
Table 4

Parameter Estimates and $\boldsymbol{d}^{\prime}$ Comparisons Obtained From Fitting an Equal-Variance, Normal Mixed-Effects Signal Detection Model to Lee and Shih's (2007) Stroop Recognition Data

\begin{tabular}{lccrc}
\hline Parameter & Estimate & $S E$ & $t$ Value & $p$ Value \\
\hline$d_{\text {nh }}^{\prime}$ & 2.197 & 0.178 & 12.33 & $<.001$ \\
$d_{\text {neg }}^{\prime}$ & 1.867 & 0.168 & 11.09 & $<.001$ \\
$d_{\text {pos }}^{\prime}$ & 1.684 & 0.166 & 10.13 & $<.001$ \\
$d_{\text {neu }}^{\prime}$ & 1.344 & 0.163 & 8.25 & $<.001$ \\
$c$ & 1.273 & 0.087 & 14.69 & $<.001$ \\
$\sigma$ & 0.563 & 0.072 & 7.79 & $<.001$ \\
$d_{\text {nh }}^{\prime}-d_{\text {neu }}^{\prime}$ & 0.853 & 0.203 & 4.20 & $<.001$ \\
$d_{\text {neg }}^{\prime}-d_{\text {neu }}^{\prime}$ & 0.524 & 0.197 & 2.66 & $<.008$ \\
$d_{\text {pos }}^{\prime}-d_{\text {neu }}^{\prime}$ & 0.341 & 0.195 & 1.74 & $<.082$ \\
\hline
\end{tabular}

Note-The symbols $d_{\text {nh }}^{\prime}, d_{\text {neg }}^{\prime}, d_{\text {pos }}^{\prime}$, and $d_{\text {neu }}^{\prime}$ indicate $d^{\prime}$ for the negative high, negative, positive, and neutral conditions, respectively. The $t$ value is for testing whether or not the corresponding parameter is equal to zero.

sponses (response), the number of stimulus presentations $(n)$, and the three indicator variables for the emotional word conditions $(\mathrm{d} 1-\mathrm{d} 3)$. For example, the neutral word condition is represented by $\mathrm{d} 1=\mathrm{d} 2=\mathrm{d} 3=0$, the negative high condition is coded by $\mathrm{d} 1=1$ and $\mathrm{d} 2=\mathrm{d} 3=0$, and so forth.

PROC NLMIXED evokes the computing procedure to be carried out for the model specified by the SAS statements. QPOINTS sets the number of quadrature points. In most cases, it is set at least to 20. Here, it is set to 25. PARMS sets the parameter starting values, which can be estimated from fitting a standard signal detection model to the data first. ETA specifies the model in which word conditions are determined by the indicator variables, the signal trials are indicated by the variable treat, and $u$ is the subject-specific criterion variable. PROBNORM specifies the cumulative standard normal distribution function. This is the link function that maps ETA to the probability of a yes response. MODEL specifies the response variable and its distribution, which is a binomial random variable in this case. RANDOM identifies the random effects and their distribution, which is normally distributed with a mean of lambda and a standard deviation of sigma. The SUBJECT = id statement identifies that the random effects vary from subject to subject. The ESTIMATE statements convert the estimated parameter values of the model to the corresponding $d^{\prime}$ value for the four emotional word conditions.

\section{Listing 1}

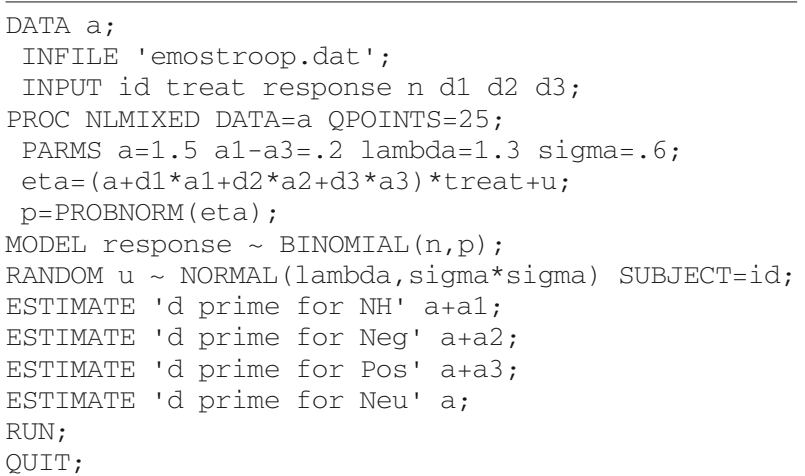

\section{Discussion}

The present method for estimating the parameters of signal detection models generalized easily to latent responses having distributions other than the normal ones. Areas under the receiver-operating characteristic curves and other performance indices for recognition performance can also be accommodated within the mixed-effects framework. To accomplish these changes, the analyst need only modify the corresponding code segment in the NLMIXED procedure to implement the new formulas. Since the statistical software SAS is widely used by researchers in industry and academia, we believe that this article contributes a novel technique for calculating signal detection theory measures beyond those described in Stanislaw and Todorov (1999). The technique proposed here should also replace current methods for calculating estimates of sensitivity from group data (Hautus, 1997) and should bring the focus back to analyses based on individual data. Furthermore, the mixed-effects signal detection models with subject-specific random effects automatically correct for extreme hit and false alarm rates without resorting to adjustments such as those advocated by Snodgrass and Corwin (1988). A condition in which the present formulation may not work well is one in which responses to trials from the same subject are correlated and the patterns of correlations vary from subject to subject. However, this is a difficult problem even when the data are rich.

Finally, by explicitly modeling changes in detectability, shifts in the locations of criteria, or interactions between the two factors as random effects and by selecting models on the basis of their ability to account for results of experimental manipulations specifically designed to induce either sensitivity changes or criterion shifts, the method may help to resolve the debate on what causes high hit and false alarm rates for generator words in the DRM paradigm (Miller \& Wolford, 1999; Wixted \& Stretch, 2000).

\section{AUTHOR NOTE}

This research was supported, in part, by Grant NSC 95-2413-H-006-017 to the first author from the National Science Council of Taiwan and Grant NSC 96-2413-H-194-011 to the second author, also from the National Science Council of Taiwan. We thank Andrew Heathcote, David Allbritton, and an anonymous reviewer for a number of helpful comments and suggestions. Correspondence concerning this article should be addressed to C.-F. Sheu, Department of Psychology, National Chung Cheng University, Min Hsiung, Chia-Yi 621, Taiwan (e-mail: psycfs@ ccu.edu.tw).

\section{REFERENCES}

Abramowitz, M., \& Stegun, I. A. (Eds.) (1972). Handbook of mathematical functions with formulas, graphs, and mathematical tables. New York: Dover.

AKaIKe, H. (1974). A new look at the statistical model identification. IEEE Transactions on Automatic Control, AC-19, 716-723.

BANKs, W. P. (1970). Signal detection theory and human memory. Psychological Bulletin, 74, 81-99.

Ben-David, B. M., Calderon, N., \& Algom, D. (2005). A signal detection analysis of the emotional Stroop effect. In J. S. Monahan, S. M. Sheffert, \& J. T. Townsend (Eds.), Fechner Day 2005 (pp. 35-38). Mt. Pleasant, MI: International Society for Psychophysics.

Davidian, M., \& Giltinan, D. M. (1995). Nonlinear models for repeated measurement data. London: Chapman and Hall/CRC Press.

DeCARLo, L. T. (1998). Signal detection theory and generalized linear models. Psychological Methods, 3, 186-205. 
Dorfman, D. D., \& Alf, E., JR. (1969). Maximum-likelihood estimation of parameters of signal-detection theory and determination of confidence intervals - Rating-method data. Journal of Mathematical Psychology, 6, 487-496.

EFron, B., \& TiBshirani, R. (1991). Bootstrap methods for standard errors, confidence intervals, and other measures of statistical accuracy. Statistical Science, 1, 54-77.

Gotlieb, I. H., \& McCAnN, C. D. (1984). Construct accessibility and depression: An examination of cognitive and affective factors. Journal of Personality \& Social Psychology, 47, 427-439.

Green, D. M., \& Swets, J. A. (1966). Signal detection theory and psychophysics. New York: Wiley.

Hautus, M. J. (1997). Calculating estimates of sensitivity from group data: Pooled versus averaged estimators. Behavior Research Methods, Instruments, \& Computers, 29, 556-562.

LEE, Y.-S., \& SHIH, P.-Y. (2007). Memory for emotional words: Effects of attention and language. Unpublished manuscript, National Chung Cheng University, Chia-Yi, Taiwan.

Luce, R. D. (1963). Detection and recognition. In R. D. Luce, R. R. Bush, \& E. Galanter (Eds.), Handbook of mathematical psychology (Vol. 1, pp. 103-189). New York: Wiley.

McCulloch, C. E., \& Searle, S. R. (2001). Generalized, linear, and mixed models. New York: Wiley.

Miller, M. B., \& Wolford, G. L. (1999). Theoretical commentary: The role of criterion shift in false memory. Psychological Review, 106, 398-405.

Murphy, V., \& Dunne, A. (2005). Mixed effects and fixed effects modelling of binary data with inter-subject variability. Journal of Pharmacokinetics \& Pharmacodynamics, 32, 245-260.

Ogilvie, J. C., \& Creelman, C. D. (1968). Maximum-likelihood estimation of receiver operating characteristic curve parameters. Journal of Mathematical Psychology, 5, 377-391.

Peterson, W. W., Birdsall, T. G., \& Fox, W. C. (1954). The theory of signal detectability. Transactions of the IRE Professional Group on Information Theory, PGIT-4, 171-212.

Pinheiro, J. C., \& Bates, D. M. (1995). Approximations to the loglikelihood function in the nonlinear mixed-effects model. Journal of Computational \& Graphical Statistics, 4, 12-35.
R Development Core Team (2006). R: A language and environment for statistical computing. Vienna: R Foundation for Statistical Computing.

Roediger, H. L., III, \& McDermott, K. B. (1995). Creating false memories: Remembering words not presented in lists. Journal of Experimental Psychology: Learning, Memory, \& Cognition, 21, 803-814.

SAS Institute, Inc. (2004). The SAS system for Unix (Version 9.1.3). Cary, NC: Author.

Schooler, L. J., \& Shiffrin, R. M. (2005). Efficiently measuring recognition performance with sparse data. Behavior Research Methods, 37, 3-10.

Schwarz, G. (1978). Estimating the dimensions of a model. Annals of Statistics, 6, 461-464.

SHEU, C.-F. (1998, August). Bayesian estimation of parameters of signal detection theory. Paper presented at the 31st Annual Mathematical Psychology Meeting, Vanderbilt University, Nashville, TN.

Sheu, C.-F., Chen, C.-T., Su, Y.-H., \& WANG, W.-C. (2005). Using SAS PROC NLMIXED to fit item response theory models. Behavior Research Methods, 37, 202-218.

Sheu, C.-F., \& HeAthrote, A. (2001). A nonlinear regression approach to estimating signal detection models for rating data. Behavior Research Methods, Instruments, \& Computers, 33, 108-114.

Snodgrass, J. G., \& Corwin, J. (1988). Pragmatics of measuring recognition memory: Applications to dementia and amnesia. Journal of Experimental Psychology: General, 117, 34-50.

Stanislaw, H., \& Todorov, N. (1999). Calculation of signal detection theory measures. Behavior Research Methods, Instruments, \& Computers, 31, 137-149.

TANNER, W. P., JR., \& Swets, J. A. (1954). A decision-making theory of visual detection. Psychological Review, 61, 401-409.

Wixted, J. T., \& Stretch, V. (2000). The case against a criterion-shift account of false memory. Psychological Review, 107, 368-376.

Wolfinger, R. D. (1999). Fitting nonlinear mixed models with the new NLMIXED procedure. In Proceedings of the 24th Annual SAS Users Group International Conference (SUGI24) (Paper 287). Cary, NC: SAS Institute.

(Manuscript received November 12, 2007; revision accepted for publication March 12, 2008.) 